\title{
THE OBLIQUE DERIVATIVE PROBLEM FOR THE HEAT EQUATION IN LIPSCHITZ CYLINDERS
}

\author{
RUSSELL M. BROWN
}

(Communicated by Barbara L. Keyfitz)

\begin{abstract}
We consider a class of initial-boundary value problems for the heat equation on $(0, T) \times \Omega$ with $\Omega$ a bounded Lipschitz domain in $\mathbf{R}^{n}$. On the lateral boundary, $(0, T) \times \partial \Omega=\Sigma_{T}$, we specify $\langle\alpha, \nabla u\rangle$ where $\nabla u$ denotes the spatial gradient of the solution and $\alpha: \Sigma_{T} \rightarrow\{x:|x|=1\}$ is a continuous vector field satisfying $\langle\alpha, \nu\rangle \geq \mu>0$ with $\nu$ the unit normal to $\partial \Omega$. On the initial surface, $\{0\} \times \Omega$, we require that the solution vanish. The lateral data is taken from $L^{p}\left(\Sigma_{T}\right)$. For $p \in(2-\epsilon, \infty)$, we show existence and uniqueness of solutions to this problem with estimates for the parabolic maximal function of the spatial gradient of the solution.
\end{abstract}

\section{INTRODUCTION}

Recently, there has been much interest in classical boundary value problems under minimal smoothness assumptions, usually Lipschitz, on the domain. In this paper, we study a class of oblique derivative problems for the heat equation in Lipschitz cylinders. Before we can state the problems we study, we will need some notation. We will use $\Omega$ to denote a bounded Lipschitz domain in $\mathbf{R}^{n}$ and we let $\Omega_{T} \equiv(0, T) \times \Omega \subset \mathbf{R} \times \mathbf{R}^{n}$ denote a Lipschitz cylinder. We use $\Sigma_{T} \equiv(0, T) \times \partial \Omega$ to denote the lateral boundary of $\boldsymbol{\Omega}_{T}$ and $\Sigma \equiv \mathbf{R} \times \partial \Omega$. We let $\alpha: \Sigma_{T} \rightarrow\left\{x \in \mathbf{R}^{n}:|x|=1\right\}$ be an oblique vector field. This means that $\langle\alpha(Q), \nu(q)\rangle \geq \mu>0$ for a.e. $Q=\left(q_{0}, q\right) \in \Sigma_{T}$. We are using $\nu$ to denote the unit inner normal to $\partial \Omega$ which points into $\Omega$ and $\langle\cdot, \cdot\rangle$ is usual inner product on $\mathbf{R}^{n}$. Throughout this paper, we will assume that $\alpha$ is uniformly continuous on $\Sigma_{T}$.

The problem we consider is

(ODP)

$$
\begin{cases}\Delta u(X)-\partial_{x_{0}} u(X)=0 & X=\left(x_{0}, x\right) \in \Omega_{T}, \\ u(0, x)=0 & x \in \Omega, \\ \partial_{\alpha} u \equiv\langle\nabla u, \alpha\rangle=f & \text { on } \Sigma_{T},\end{cases}
$$

Received by the editors November 18, 1988 and, in revised form, February 7, 1989.

1980 Mathematics Subject Classification (1985 Revision). Primary 35K20.

Key words and phrases. Heat equation, initial-boundary value problems, nonsmooth domains.

Supported by an NSF postdoctoral fellowship. This research was conducted while the author was in residence at the Mathematical Sciences Research Institute. 
where $\nabla=\left(\partial_{x_{1}}, \ldots \partial_{x_{n}}\right)$ denotes the spatial gradient and $\Delta=\sum_{i=1}^{n} \partial_{x_{i}}^{2}$ is the Laplacian in the spatial variables. We take the datum $f$ from $L^{p}\left(\Sigma_{T}\right)$; thus we must specify what is meant by the restriction of $\nabla u$ to $\Sigma_{T}$.

To do this, we first define the parabolic metric,

$$
\delta(X ; Y)=|x-y|+\left|x_{0}-y_{0}\right|^{1 / 2}
$$

where $X=\left(x_{0}, x\right)$ and $Y$ lie in $\mathbf{R} \times \mathbf{R}^{n}$. For a set $E \subset \mathbf{R} \times \mathbf{R}^{n}$, we let $\delta(X ; E)$ denote the distance between $X$ and $E$ :

$$
\delta(X ; E)=\inf _{Y \in E} \delta(X ; Y) .
$$

For $\gamma>0$ and $P=\left(p_{0}, p\right) \in \Sigma_{T}$, we let

$$
\Gamma(P)=\Gamma(P, \gamma)=\left\{Y: \delta(P ; Y)<(1+\gamma) \delta(Y ; \Sigma) \text { and } y_{0} \leq p_{0}\right\}
$$

denote the parabolic cone with vertex at $P$. We will generally view $\gamma$ as fixed and omit the dependence of $\Gamma(P)$ on $\gamma$. Finally, we remark that we will assume that $\gamma$ is large enough so that $P+s \alpha(P) \in \Gamma(P)$ for $s$ sufficiently small. Now we may define the restriction of $\nabla u$ to $\Sigma_{T}$ via parabolic limits:

$$
\nabla u(P) \equiv \lim _{\Gamma(P) \ni Y \rightarrow P} \nabla u(Y)
$$

Our estimates for solutions to (ODP) will be given in terms of the parabolic maximal function. For a function $v$ defined on $\Omega_{T}$, the parabolic maximal function of $v, N(v)$, is given by

$$
N(v)(P)=\sup _{Y \in \Gamma(P)}|v(Y)|, \quad P \in \Sigma_{T} .
$$

We assume that $v$ has been defined to be zero for $y_{0}<0$. With this preparation, we may state our result for (ODP):

Theorem. Let $\Omega$ be a Lipschitz domain and let $\alpha$ be a continuous oblique vector field on $\Sigma_{T}$. There exists $\epsilon>0$ such that for $f \in L^{p}\left(\Sigma_{T}\right), 2-\epsilon<p<\infty$, (ODP) has a unique solution which satisfies

$$
\|N(\nabla u)\|_{L^{p}\left(\Sigma_{T}\right)} \leq C\|f\|_{L^{p}\left(\Sigma_{T}\right)} .
$$

The constant $C$ depends on $p, \gamma, \mu, T$, the modulus of continuity of $\alpha$ and the domain $\Omega$. The value of $\epsilon$ depends only on the Lipschitz constant of $\Omega$.

Remark. The requirement that $N(\nabla u) \in L^{p}\left(\Sigma_{T}\right)$, forces the existence of the parabolic limits of $\nabla u$. For $p \in(2-\epsilon, \infty]$, this follows from the work of Fabes and Salsa on the initial-Dirichlet problem [FS]. We shall see that the range of $p$ 's for which we can solve (ODP) is the same as the range of $p$ 's for which we can solve the initial-Dirichlet problem.

The range $2-\epsilon<p<\infty$ is sharp. To be precise, given a $p<2$, we may find a Lipschitz domain and a continuous oblique vector field such that the estimate of the Theorem cannot hold. To see this, suppose that $\alpha=e_{n}$ on a 
cube $I_{2 r}(P) \subset \Sigma_{T}$. Restricting our attention to data supported in $I_{r}(P)$, the Theorem gives the estimate

$$
\left\|N\left(\partial_{x_{n}} u\right)\right\|_{L^{p}\left(I_{2 r}(P)\right)} \leq C\left\|\partial_{x_{n}} u\right\|_{L^{p}\left(I_{r}(P)\right)}
$$

for the caloric function $\partial_{x_{n}} u$ and all $f \in L^{p}\left(I_{r}(P)\right)$. (We use the term caloric function to refer to a solution of the heat equation.) However, it is known that given $p<2$, one may find a Lipschitz domain for which the density of caloric measure with respect to surface measure does not lie in $L^{p^{\prime}}, 1 / p+1 / p^{\prime}=1$. Hence, the above estimate cannot hold. It is also well known that the Theorem fails for $p=\infty$ even when the domain is a half space and $\alpha$ is constant.

This theorem, for $p=2$ and time independent vector fields, first appeared in the author's thesis [B2]. A different proof is given there and it is also shown that the solutions may be represented as single-layer heat potentials. We remark that one may establish the representation of solutions of (ODP) for $p$ near 2 using the estimates of our Theorem and the estimates from [B3]. These tools allow one to carry out the familiar argument of G. Verchota. However, we will not give any additional details here.

G. Lieberman has obtained results for (ODP) when the data lies in Hölder classes and then the vector field $\alpha$ must be Hölder continuous. He also considers more general second order parabolic operators and allows inhomogeneous initial data and forcing terms.

A.P. Calderón has studied the analogue of (ODP) for Laplace's equation in [C] and obtained estimates similar to ours when $p$ is near 2. Kenig and Pipher extended Calderón's result to $p$ in the range $2-\epsilon<p<\infty$ [KP]. The main techniques used here and in Kenig and Pipher's work are similar, but certain technical complications arise in the elliptic case which are not present for the heat equation.

We defer discussion of the proof of the Theorem until $\S 1$ where we will give three lemmas which are the main steps in the proof of our theorem. $\S \S 2$ to 4 are devoted to the proofs these lemmas. In $\S 5$, we complete the proof of our theorem.

We will continue to use the notation given in this introduction. Additional notation will be introduced as needed. We will let $c$ and $C$ denote constants whose numerical value may change from line to line. In the statement of each result, we will list the parameters that constants appearing in that result are allowed to depend on. We will use $X$ and $Y$ to denote points in $\Omega_{T}$ and $P$ and $Q$ for points on the lateral boundary $\Sigma_{T}$. We will write $X=\left(X^{\prime}, x_{n}\right)=\left(x_{0}, x\right)=\left(x_{0}, x^{\prime}, x_{n}\right)$ when we wish to distinguish the last variable, the time variable or both. Similarly, we will write $P=\left(p_{0}, p\right)$ for points on the boundary.

We recall some well-known interior estimates which are satisfied by caloric 
functions. For $X \in \mathbf{R} \times \mathbf{R}^{n}$ and $r>0$, let $J_{r}(X)=\left\{Y: \delta(X ; Y)<r\right.$ and $y_{0}<$ $\left.x_{0}\right\}$. Then for $u$, a caloric function on $J_{2 r}(X)$, we have

$$
\sup _{Y \in J_{r}(X)}\left|\partial_{y}^{\alpha} \partial_{y_{0}}^{\beta} u(Y)\right| \leq C_{\alpha, \beta, p} r^{-2 \beta-|\alpha|}\left(\frac{1}{r^{n+2}} \int_{J_{2 r}(X)}|u(Y)|^{p} d Y\right)^{1 / p} .
$$

where $\alpha=\left(\alpha_{1}, \ldots, \alpha_{n}\right)$ is a multi-index, $\beta$ is a nonnegative integer and $1 \leq$ $p \leq \infty$ (with the obvious interpretation when $p=\infty$ ).

Finally, I would like to thank the referee for pointing out the relevance of Trudinger's work in the proof of Lemma 1.2.

\section{Three LemMas}

Throughout this paper, we will use $\alpha$ to denote a uniformly continuous oblique vector field as defined in the Introduction and $\Omega$ will be a bounded Lipschitz domain, a precise definition is given after Lemma 1.1.

The main step in the proof of our Theorem is the study of a model problem. In the model, we simplify the situation by assuming that the domain is given by $D=\left\{\left(x^{\prime}, x_{n}\right): x_{n}>\phi\left(x^{\prime}\right)\right\}$ where $\phi: \mathbf{R}^{n-1} \rightarrow \mathbf{R}$ is a Lipschitz function with $\|\nabla \phi\|_{\infty} \leq m$ and that the oblique vector field is constant. In fact, we may assume that $\alpha$ is $e_{n}$, the unit coordinate vector in the $x_{n}$-direction.

Our first lemma gives estimates for this model problem. We will use $D_{\infty}$ to denote the cylinder $\mathbf{R} \times D$ and we let $S=\mathbf{R} \times \partial D$ denote the lateral boundary of $D_{\infty}$.

Lemma 1.1. There exists $\epsilon>0$ such that if $f \in L^{p}(S), p \in(2-\epsilon, \infty)$, then there exists a function $u$, caloric on $D_{\infty}$, with $\partial_{x_{n}} u=f$ and

$$
\|N(\nabla u)\|_{L^{p}(S)} \leq C\|f\|_{L^{p}(S)} .
$$

The constant $C$ depends on $m, p$ and $\gamma$. The value of $\epsilon$ depends only on $m$.

In approximating (ODP) by the model problem discussed in Lemma 1.1, there will be two sources of error: 1) The direction $\alpha$ in our boundary operator is not constant. 2) We want to study (ODP) on a bounded Lipschitz domain instead of the graph domain used in Lemma 1.1. Since we are assuming that $\alpha$ is continuous, it will be 'almost constant' on small bits of $\Sigma_{T}$, thus the first source of error will be fairly easy to handle. The second source of error will be handled using the next lemma. Before stating this lemma, we need to give a precise definition of a bounded Lipschitz domain and a few related objects.

Let $m>0$ and $r_{0}>0$ be given and set $Z=\left\{\left(x^{\prime}, x_{n}\right):\left|x_{i}\right|<r_{0}, i=\right.$ $1, \ldots, n-1$ and $\left.x_{n}<100 n(1+m) r_{0}\right\}$ and let $\phi: \mathbf{R}^{n-1} \rightarrow\left(-m r_{0}, m r_{0}\right)$ be a continuous function. We say that $(Z, \phi)$ is a coordinate cylinder for $\Omega$, a bounded open set in $\mathbf{R}^{n}$, if

$$
\begin{gathered}
\Omega \cap 100 Z=\left\{\left(x^{\prime}, x_{n}\right): x_{n}>\phi\left(x^{\prime}\right)\right\} \cap 100 Z \text { and } \\
\partial \Omega \cap 100 Z=\left\{\left(x^{\prime}, x_{n}\right): x_{n}=\phi\left(x^{\prime}\right)\right\} \cap 100 Z
\end{gathered}
$$


where $100 Z$ denotes the dilation of $Z$ by a factor of 100 . We say that $\Omega$ is a Lipschitz domain if there exists a covering of $\partial \Omega$ by coordinate cylinders $\left\{\left(Z_{i}, \phi_{i}\right)\right\}$ such that the functions $\phi_{i}$ satisfy $\left\|\nabla \phi_{i}\right\|_{\infty} \leq m$. The coordinate systems used to define each coordinate cylinder are allowed to vary by a rigid motion.

Let $P=\left(x_{0}, x^{\prime}, \phi_{i}\left(x^{\prime}\right)\right)$ be a point on $\Sigma \cap\left(\mathbf{R} \times Z_{i}\right)$. We define a surface cube with 'center' $P$ and sidelength $r$ by

$$
\begin{aligned}
I_{r}(P)=\left\{\left(y_{0}, y^{\prime}, \phi_{i}\left(y^{\prime}\right)\right): x_{0}-r^{2}<y_{0}\right. & <x_{0} \\
& \text { and } \left.\left|x_{i}-y_{i}\right|<r, i=1, \ldots, n-1\right\} .
\end{aligned}
$$

We also define local domains $\Psi_{r}(P)$ near the boundary. These are given by

$$
\begin{aligned}
\Psi_{r}(P)=\left\{\left(y_{0}, y^{\prime}, y_{n}\right): x_{0}-r^{2}<y_{0}<x_{0},\left|x_{i}-y_{i}\right|<r, i\right. & =1, \ldots, n-1 \\
\text { and } \phi_{i}\left(y^{\prime}\right) & \left.<y_{n}<\phi_{i}\left(y^{\prime}\right)+r\right\}
\end{aligned}
$$

where again $P=\left(x_{0}, x^{\prime}, x_{n}\right)$. Observe that $\Psi_{r}(P)$ and $I_{r}(P)$ may only be defined when $r$ is small, say $r<r_{0}$. If $P$ lies in several coordinate cylinders, then we have several choices for $\Psi_{r}(P)$. We ignore this ambiguity since our estimates will hold for any choice. However, we do assume that the choices are made consistently so that $\overline{I_{r}(P)}=\overline{\Psi_{r}(P)} \cap \Sigma$.

To state the next lemma, we define a truncated parabolic maximal function, $N_{r}(v)(P) \equiv \sup _{Y \in \Gamma_{r}(P)}|v(Y)|$ where $\Gamma_{r}(P)=\Gamma(P) \cap\{Y: \delta(Y ; P)<r\}$.

Lemma 1.2. Let $u$ be caloric on $\Psi_{32 r}(Q)$, suppose that $\partial_{x_{n}} u=0$ a.e. on $I_{32 r}(Q)$ and that $N_{r}\left(\partial_{x_{n}} u\right) \in L^{p}\left(I_{16 r}(Q)\right)$ for some $p \in(2-\epsilon, \infty)$. Then we have the estimate

$$
\sup _{\Psi_{2 r}(Q)}|\nabla u(X)| \leq C\left(\frac{1}{r^{n+2}} \int_{\Psi_{16 r}(Q)}|\nabla u(Y)|^{p} d Y\right)^{1 / p} .
$$

As a trivial consequence, we have

$$
\int_{I_{r}(Q)} N_{r}(\nabla u)(Q)^{p} d Q \leq C r^{-1} \int_{\Psi_{16 r}(Q)}|\nabla u(Y)|^{p} d Y .
$$

The constant $C$ depends only on $m, \gamma$ and $p$ and $\epsilon>0$ depends only on $m$.

Lemma 1.2 may be proved using the Hölder estimates for (ODP) given in [L2, Lemma 14.1]. However, some work is required to show that $u$ satisfies the a priori estimates of this Lemma. Since a direct proof is available, we give the argument in $\S 3$.

Finally, using Lemma 1.1 and 1.2, we may prove an a priori estimate for (ODP). With this estimate established, the proof of our Theorem will be fairly routine. In the statement of our third lemma, we use $\Omega_{\eta}$ to denote $\{x \in$ $\Omega: \operatorname{dist}(x, \partial \Omega)>\eta\}$ and let $\Omega_{\eta, T}=(0, T) \times \Omega_{\eta}$. 
Lemma 1.3. Suppose that $u$ is caloric in $\Omega_{T}, u(0, x)=0$ and $N(\nabla u) \in$ $L^{p}\left(\Sigma_{T}\right), p \in(2-\epsilon, \infty)$, then $u$ satisfies

$$
\int_{\Sigma_{T}} N(\nabla u)(Q)^{p} d Q \leq C\left(\int_{\Sigma_{T}}\left|\partial_{\alpha} u(Q)\right|^{p} d Q+\int_{\Omega_{\eta, T}}|\nabla u(Y)|^{p} d Y\right) .
$$

The values of $C$ and $\eta$ depend only on $\mu, p, m$, the diameter of $\Omega$, the modulus of continuity of $\alpha$ and the maximum overlap of the coordinate cylinders $\left\{100 Z_{i}\right\}$. The value of $\epsilon$ is determined by $\mathrm{m}$.

\section{ESTIMATES FOR THE MODEL PROBLEM}

Lemma 1.1 is essentially a statement about the Dirichlet problem. The proof of this lemma consists of two steps. The first step is to construct a caloric function $v=\partial_{x_{n}} u$ which satisfies $N(v) \in L^{p}(S)$ and $v=f$ on $S$, that is solve the Dirichlet problem. From $v$, we may easily construct $\partial_{x_{0}} u, \ldots, \partial_{x_{n-1}} u$ and hence $u$. To show that $N\left(\partial_{x_{i}} u\right) \in L^{p}(S)$ for $i=1, \ldots n-1$, we use an area integral. For a smooth function $v$ on $D_{\infty}$, the area integral of $v$ is defined by

$$
A_{\gamma}(v)(P)^{2}=\int_{\Gamma(P, \gamma)}|\nabla v(Y)|^{2} \delta(Y ; S)^{-n} d Y, \quad P \in S .
$$

It is known that if $v$ is caloric in $D_{\infty}$ and vanishes at infinity, then the parabolic maximal function of $v$ is in $L^{p}(S)$ if and only if the area integral of $v$ lies in $L^{p}(S)$. Finally, a lemma of E.M. Stein gives us pointwise control of $A_{\gamma}\left(\partial_{x_{i}} u\right)$ in terms of $A_{C \gamma}\left(\partial_{x_{n}} u\right)$. Combining these observations gives Lemma 1.1. We now state the needed results and give a few additional details to complete the proof outlined above.

The Dirichlet problem for the heat equation in Lipschitz cylinders was studied by Fabes and Salsa. We quote their result as:

Theorem 2.1 [FS, Theorem 3.2]. There exists $\epsilon>0$ such that for $f \in L^{p}(S)$, $p \in(2-\epsilon, \infty]$, there exists $v$ on $D_{\infty}$ which satisfies

$$
\begin{cases}\Delta v-\partial_{x_{0}} v=0 & \text { in } D_{\infty}, \\ v=f & \text { on } S, \\ \|N(v)\|_{L^{p}(S)} \leq C\|f\|_{L^{p}(S)} . & \end{cases}
$$

The constant $C$ depends only on $p$ and $m$. The value of $\epsilon$ depends on on $m$. Remark 1. Fabes and Salsa only treat the case when $p \in[2, \infty]$. The extension to $2-\epsilon<p<2$ follows from their estimates (see (3.1) of [FS]) and the techniques of Coifman and Fefferman in [CF].

Remark 2. Fabes and Salsa only treat the case of bounded domains. The extension to unbounded domains is not particularly difficult. Another proof is given in [B3] which discusses the case of graph domains.

Next, we give our result relating $A_{\gamma}(v)$ and $N(v)$. 
Proposition 2.2 [B2, Corollary to Theorem 4.3]. Let $v$ be caloric in $D_{\infty}$ and suppose that $\lim _{i \rightarrow \infty} \sup _{\delta(X ; S)>r}|v(X)|=0$. Then for $p \in(0, \infty)$ we have

$$
C^{-1}\|N(v)\|_{L^{p}(S)} \leq\left\|A_{\gamma}(v)\right\|_{L^{p}(S)} \leq C\|N(v)\|_{L^{p}(S)} .
$$

The constant $C$ depends only on $p, m$ and $\gamma$.

Remark 1. Again the case of graph domains is not treated in [B2]. However, the proof for graph domains is essentially the same as that given in [B2].

Remark 2. It is well known that area integrals or parabolic maximal functions defined using different cone openings have comparable $L^{p}$-norms. See [T, pp. 314-317 and 367]. Thus $\gamma$ plays no essential role in this proposition.

Remark 3. The paper [B2] as well as most previous work on the heat equation uses cones defined by $\widetilde{\Gamma}(p, \gamma)=\left\{Y \in D_{\infty}: \delta(Y ; P)<(1+\gamma) \delta(Y ; S)\right\}$ while the cones in this paper were defined by $\Gamma(P, \gamma)=\widetilde{\Gamma}(p, \gamma) \cap\left\{Y: y_{0}<p_{0}\right\}$. The above proposition is true for either family of cones. In fact arguments similar to those in [T, pp. 314-317 and 367] can be used to show that the $L^{p}(S)$-norms of area integrals defined using either family of cones are comparable and similarly for parabolic maximal functions. The reader who prefers not to verify this may simply assume that in this section $N(v)$ and $A_{\gamma}(v)$ are formed using the full cones $\widetilde{\Gamma}(P, \gamma)$. The truth of Lemma 1.1 for the parabolic maximal function formed using these larger cones trivially implies the truth of Lemma 1.1 for the smaller cones.

The one-sided cones $\Gamma(P)$ were not chosen simply for the joy of changing notation. There are two reasons to prefer them. 1) These cones reflect the evolutive nature of the heat equation. In particular, the parabolic maximal function formed using the cones $\Gamma(P)$ has the property that $N(v)(P)$ depends only on the behavior of the solution at times $\left.y_{0} \leq p_{0} .2\right)$ Using the cones $\Gamma(P)$ simplifies the arguments used to prove Lemma 1.3.

Finally, we recall a lemma of E. M. Stein. Though he only considers harmonic functions, the caloric case is identical.

Lemma 2.3 [S, pp. 213-216]. Let $\nabla u$ be the gradient of a caloric function in $D_{\infty}$. Then for a suitable constant, we have

$$
A_{\gamma}\left(\partial_{x_{i}} u\right)(P) \leq C A_{C \gamma}\left(\partial_{x_{n}} u\right)(P), \quad P \in S .
$$

The constant depends on $\gamma$ and $m$.

With these results in hand, we may quickly finish the proof of Lemma 1.1.

Proof of Lemma 1.1. Using Theorem 2.1, we construct $u_{n}$, the solution of the Dirichlet problem in $D_{\infty}$ with datum $f$. For $i=0, \ldots, n-1$, we set

$$
u_{i}\left(X^{\prime}, x_{n}\right)=-\int_{x_{n}}^{\infty} \partial_{x_{i}} u_{n}\left(X^{\prime}, s\right) d s, \quad\left(X^{\prime}, x_{n}\right) \in D_{\infty} .
$$


Using interior estimates, it is easy to see that

$$
\begin{gathered}
\left|\partial_{x_{i}} u_{n}(X)\right| \leq C\left\|N\left(u_{n}\right)\right\|_{L^{p}(S)} \delta(X ; S)^{-1-\frac{n+1}{p}}, \quad i=1, \ldots, n-1 \\
\text { and }\left|\partial_{x_{0}} u_{n}(X)\right| \leq C\left\|N\left(u_{n}\right)\right\|_{L^{p}(S)} \delta(X ; S)^{-2-\frac{n+1}{p}} .
\end{gathered}
$$

Hence, the integrals defining $u_{i}$ are absolutely convergent. Also one may verify that $\partial_{x_{i}} u_{j}=\partial_{x_{j}} u_{i}$ for $i, j=0, \ldots, n$ and, since $u_{n}$ is caloric, that $u_{0}=$ $\sum_{i=1}^{n} \partial_{x_{i}} u_{i}$. Since $D_{\infty}$ is simply connected, there exists a caloric function $u$ such that $\left(\partial_{x_{0}} u, \nabla u\right)=\left(u_{0}, u_{1}, \ldots, u_{n}\right)$. From Lemma 2.3 and Proposition 2.2 , it follows that

$$
\|N(\nabla u)\|_{L^{p}(S)} \leq C\left\|N\left(u_{n}\right)\right\|_{L^{p}(S)}
$$

and Theorem 2.1 gives

$$
\left\|N\left(u_{n}\right)\right\|_{L^{p}(S)} \leq C\|f\|_{L^{p}(S)}
$$

Combining these observations establishes the estimate of Lemma 1.1.

\section{Proof of Lemma 1.2}

By a parabolic rescaling, we may assume that $r=1$. We begin by studying $\partial_{x_{n}} u$. Our hypothesis that $N_{1}\left(\partial_{x_{n}} u\right) \in L^{p}\left(I_{16}(Q)\right)$ is sufficient to guarantee uniqueness in the Dirichlet problem (see [FS, Theorem 3.2]). Hence it follows that $\partial_{x_{n}} u$ vanishes continuously on $I_{16}(Q)$. In fact, we have the stronger estimate

$$
\left|\partial_{x_{n}} u(X)\right| \leq C \delta(X ; \Sigma)^{\beta} \sup _{Y \in \Psi_{8}(Q)}\left|\partial_{x_{n}} u(Y)\right|, \quad X \in \Psi_{4}(Q) .
$$

Recall that (3.1) may be established using the maximum principle and rescaling (see [JK, Lemma 5.1] for the corresponding result for harmonic functions). Given $X \in \Psi_{2}(Q)$, let $\rho=\delta(X ; \Sigma)$ and observe that there exists a constant $c$ such that $J_{c \rho}(X) \subset \Psi_{4}(Q)$ and $\delta\left(J_{c \rho}(X) ; \Sigma\right) \geq c \rho$. From interior estimates for caloric functions, it follows that

$$
\begin{aligned}
\left|\nabla \partial_{x_{n}} u(X)\right| \leq C \rho^{-1} & \sup _{Y \in J_{c p}(X)}\left|\partial_{x_{n}} u(Y)\right| \\
& \leq C^{\prime} \delta(X ; \Sigma)^{\beta-1} \sup _{Y \in \Psi_{8}(Q)}\left|\partial_{x_{n}} u(Y)\right|, \quad X \in \Psi_{2}(Q) .
\end{aligned}
$$

The second inequality uses (3.1). Let $X=\left(X^{\prime}, \phi\left(x^{\prime}\right)+t\right) \in \Psi_{2}(Q)$. We have

$$
\begin{aligned}
& \left|\nabla u\left(X^{\prime}, \phi\left(x^{\prime}\right)+t\right)\right| \\
& \leq \int_{t}^{2}\left|\nabla \partial_{x_{n}} u\left(X^{\prime}, \phi\left(x^{\prime}\right)+s\right)\right| d s+\sup _{P \in I_{2}(Q)}\left|\nabla u\left(P+2 e_{n}\right)\right| \\
& \quad \leq C \int_{0}^{2} s^{\beta-1} d s \cdot \sup _{Y \in \Psi_{8}(Q)}\left|\partial_{y_{n}} u(Y)\right|+\sup _{P \in I_{2}(Q)}\left|\nabla u\left(P+2 e_{n}\right)\right| .
\end{aligned}
$$


Since the integral in this last expression is finite, our lemma will follow from (3.3) once we prove the following estimates

$$
\sup _{P \in I_{2}(Q)}\left|\nabla u\left(P+2 e_{n}\right)\right| \leq C\left(\int_{\Psi_{16}(Q)}|\nabla u(Y)|^{p} d Y\right)^{1 / p}
$$

and

$$
\sup _{Y \in \Psi_{8}(Q)}\left|\partial_{y_{n}} u(Y)\right| \leq C\left(\int_{\Psi_{16}(Q)}\left|\partial_{y_{n}} u(Y)\right|^{p} d Y\right)^{1 / p}
$$

The first is estimate, (3.4), is contained in the interior estimates given in the Introduction. The second estimate may be proven using Moser's iteration scheme. See [Tr, Theorem 4.3].

\section{Proof of Lemma 1.3}

We observe that if we set $u=0$ for $x_{0}<0$, then $u$ is a caloric function on $(-\infty, T) \times \Omega$. We will use this fact in applying Lemma 1.2 since dilates of the domains $\Psi_{r}(P) \subset \Omega_{T}$ may extend into the region $x_{0}<0$.

We begin by claiming the following estimate for $p \in(2-\epsilon, \infty)$ and $r$ sufficiently small:

$$
\begin{gathered}
\int_{I_{r}(P)} N_{r}(\nabla u)(Q)^{p} d Q \leq C\left(\int_{I_{32 r}(P)}\left|\partial_{\alpha} u(Q)\right|^{p}+\omega(C r)^{p}|\nabla u(Q)|^{p} d Q\right. \\
\left.+r^{-1} \int_{\Psi_{32 r}(P)}|\nabla u(Y)|^{p} d Y\right) .
\end{gathered}
$$

In the above estimate,

$$
\omega(r)=\sup \{|\alpha(P)-\alpha(Q)|: \delta(P ; Q)<r\}
$$

denotes the modulus of continuity of $\alpha$.

Assuming the estimate (4.1), we complete the proof of Lemma 1.3. For each $r, 0<r<r_{0}$, we may choose a covering of $\Sigma_{T}$ by cubes $\left\{I_{r}\left(P_{i}\right)\right\}$ which satisfy $I_{r}\left(P_{i}\right) \subset \Sigma_{T}$ and

$$
\sum_{i} \chi_{\Psi_{32 r}\left(P_{i}\right)} \leq C
$$

Applying (4.1) on each cube $I_{r}\left(P_{i}\right)$ and summing, we obtain

$$
\begin{aligned}
\int_{\Sigma_{T}} N_{r}(\nabla u)(Q)^{p} d Q \leq C\left(\int_{\Sigma_{T}}\left|\partial_{\alpha} u(Q)\right|^{p}+\omega(C r)^{p}|\nabla u(Q)|^{p} d Q\right. & \\
& \left.+r^{-1} \int_{\Omega_{T}}|\nabla u(Y)|^{p} d Y\right) .
\end{aligned}
$$

Since $\alpha$ is continuous, we may choose $r$ small so that $C \omega(C r)^{p}=\frac{1}{2}$ and absorb the second term in the integral over $\Sigma_{T}$ into the lefthand side of (4.2). Next we observe that we may use interior estimates to establish

$$
\int_{\Sigma_{r}} N(\nabla u)(Q)^{p} d Q \leq \int_{\Sigma_{T}} N_{r}(\nabla u)(Q)^{p} d Q+C_{r} \int_{\Omega_{T}}|\nabla u(Y)|^{p} d Q .
$$


Finally, it is easy to see that

$$
\int_{\Omega_{T} \backslash \Omega_{\eta, T}}|\nabla u(Y)|^{p} d Y \leq C \eta \int_{\Sigma_{T}} N(\nabla u)(Q)^{p} d Q .
$$

Combining these last three inequalities and choosing $\eta$ small completes the proof of the lemma.

We turn to the proof of (4.1). We fix a cube $I_{r}(P)$ and choose a coordinate system in a neighborhood of $I_{32 r}(P)$ so that $e_{n}=\alpha(P)$ and $\partial \Omega=\left\{x_{n}=\right.$ $\left.\phi\left(x^{\prime}\right)\right\}$. Observe that the Lipschitz constant of $\phi$ is determined by $\inf \langle\alpha, \nu\rangle$ and the Lipschitz constant for $\Omega$. We let $f=\partial_{x_{n}} u \cdot \chi_{I_{32 r}(P)}$ and view $f$ as a function on the graph $\left\{x_{n}=\phi\left(x^{\prime}\right)\right\} \equiv \partial D$. We let $v$ be the solution of the model problem with data $f$ on the graph domain $D_{\infty}$. On $\Psi_{32 r}(P)$, we set $w=u-v$. We have

$$
\int_{I_{r}(P)} N_{r}(\nabla u)(Q)^{p} d Q \leq C \int_{I_{r}(P)} N(\nabla v)(Q)^{p}+N_{r}(\nabla w)(Q)^{p} d Q .
$$

Using Lemma 1.1 and recalling the definition of $f$ and $e_{n}$, we obtain

$$
\begin{aligned}
\int_{I_{r}(P)} N(\nabla v)(Q)^{p} d Q & \leq C \int_{I_{32 r}(P)}\left|\partial_{x_{n}} u(Q)\right|^{p} d Q \\
& \leq C \int_{I_{32 r}(P)}\left|\partial_{\alpha} u(Q)\right|^{p}+\omega(C r)^{p}|\nabla u(Q)|^{p} d Q .
\end{aligned}
$$

Since $\partial_{x_{n}} w$ vanishes on $I_{32 r}(P)$, we may use Lemma 1.2 to see that $\nabla w$ is bounded on $\Psi_{2 r}(P)$ and using the estimate from Lemma 1.2, we obtain

$$
\begin{aligned}
\int_{I_{r}(P)} N_{r}(\nabla w)(Q)^{p} d Q & \leq C r^{-1} \int_{\Psi_{16 r}(Q)}|\nabla w(Q)|^{p} d Q \\
& \leq C^{\prime} r^{-1} \int_{\Psi_{16 r}(Q)}|\nabla u(Q)|^{p}+|\nabla v(Q)|^{p} d Q
\end{aligned}
$$

where we have used $\nabla w=\nabla u-\nabla v$ for the second inequality. Now from the $L^{p}$-estimate for $N(\nabla v)$ in Lemma 1.1 and the procedure used to establish (4.3) we obtain

$$
\text { (4.5) } \begin{aligned}
\int_{\Psi_{16 r}(P)}|\nabla v(Q)|^{p} d Q & \leq C \int_{S} N(\nabla v)(Q)^{p} d Q \\
& \leq C^{\prime}\left(\int_{I_{32 r}(P)}\left|\partial_{\alpha} u(Q)\right|^{p}+\omega(C r)^{p}|\nabla u(Q)|^{p} d Q\right) .
\end{aligned}
$$

Combining (4.3) to (4.5) gives (4.1).

4.6 Corollary to Lemma 1.3. Let $u$ and $p$ be as in Lemma 1.3. Then there exists $T_{0}$ such that for $T \leq T_{0}$,

$$
\int_{\Sigma_{I}} N(\nabla u)(Q)^{p} d Q \leq C \int_{\Sigma_{T}}\left|\partial_{\alpha} u(Q)\right|^{p} d Q .
$$


The constant $C$ and $T_{0}$ depend on $\gamma, m, p, \mu$, the modulus of continuity of $\alpha$ and $\left\{Z_{i}\right\}$.

Proof. We wish to show that

$$
\int_{\Omega_{\eta, T}}|\nabla u(Y)|^{p} d Y \leq C_{\eta} T^{p} \int_{\Sigma_{T}} N(\nabla u)(Q)^{p} d Q .
$$

Then we use this inequality to bound the last term of the estimate in Lemma 1.3 and choose $T_{0}$ small which establishes the Corollary.

To establish (4.7), recall that $\gamma$ is the parameter used to define the cones for $N(\cdot)$. Applying interior estimates we see that if $Y \in \Gamma(Q, \gamma / 2)$ then $\left|\partial_{y_{0}} \nabla u(Y)\right| \leq C \delta(Y ; \Sigma)^{-2} N(\nabla u)(Q)$. Hence

$$
\int_{\Omega_{\eta, T}}\left|\partial_{y_{0}} \nabla u(Y)\right|^{p} d Y \leq C \eta^{-2} \int_{\Sigma_{T}} N(\nabla u)(Q)^{p} d Q
$$

with $C$ independent of $T$. Next we observe that since $\nabla u(0, y)=0$, we have

$$
\int_{\Omega_{\eta, T}}|\nabla u(Y)|^{p} d Y \leq T^{p} \int_{\Omega_{\eta, T}}\left|\partial_{y_{0}} \nabla u(Y)\right|^{p} d Y
$$

Combining these observations gives (4.7).

\section{Proof of The THeOReM}

In this section, we complete the proof of the Theorem. We begin by establishing the uniqueness assertion in the Theorem.

Proposition 5.1. Let $\epsilon$ be as in Lemma 1.3. Let $u$ be caloric, zero initially and satisfy $N(\nabla u) \in L^{p}\left(\Sigma_{T}\right)$, for some $p \in(2-\epsilon, \infty)$. If $\partial_{\alpha} u=0$ a.e. on $\Sigma_{T}$, then $u \equiv 0$.

Proof. Using Corollary 4.6, we see that $\|N(\nabla u)\|_{L^{p}\left(\Sigma_{T_{0}}\right)}=0$ for some small $T_{0}>0$. Since $u(0, x)=0$ and $u$ is caloric, it follows that $u \equiv 0$ on $\Sigma_{T_{0}}$. Repeating this argument on $\left(T_{0}, 2 T_{0}\right) \times \Omega$ we conclude that $u \equiv 0$ on $\Omega_{2 T_{0}}$. By iteration, it follows that $u=0$ on $\Omega_{T}$ for any $T$.

Our next result shows that we may omit the term involving the $L^{p}\left(\Omega_{\eta, T}\right)$ norm of $\nabla u$ on the righthand side of the estimate in Lemma 1.3. The argument is fairly standard, but is included to show that the constant in the resulting estimate depends on the vector field $\alpha$ only through the modulus of continuity of $\alpha$ and the lower bound for $\langle\alpha, \nu\rangle$.

Proposition 5.2. Suppose that $u$ is caloric on $\Omega_{T}, u(0, x)=0$ and $N(\nabla u) \in$ $L^{p}\left(\Sigma_{T}\right), p \in(2-\epsilon, \infty)$. Then there exists a constant $C$ depending only on $\mu$, $p, \Omega, T$ and the modulus of continuity of $\alpha$ such that

$$
\|N(\nabla u)\|_{L^{p}\left(\Sigma_{T}\right)} \leq C\left\|\partial_{\alpha} u\right\|_{L^{p}\left(\Sigma_{T}\right)} .
$$


Proof. We fix a modulus of continuity $\omega, \mu>0, \Omega, T$ and $p \in(2-\epsilon, \infty)$. Suppose that the desired estimate does not hold and thus there exists a sequence of vector fields $\alpha_{i}$ and caloric functions $u_{i}$ such that

$$
\left\|\partial_{\alpha_{i}} u_{i}\right\|_{L^{p}\left(\Sigma_{T}\right)} \rightarrow 0 \text { as } i \rightarrow \infty
$$

but

$$
\int_{\Omega_{\eta, T}}\left|\nabla u_{i}(Y)\right|^{p} d Y=1 \quad \text { for each } i .
$$

The family $\left\{\alpha_{i}\right\}$ is equicontinuous on $\overline{\Sigma_{T}}$, hence we may choose a subsequence (which we still call $\left\{\alpha_{i}\right\}$ ) that converges uniformly to a continuous oblique vector field $\alpha_{0}$. By refining our sequence again, we may guarantee that $u_{i}$ and all its derivatives converge to a caloric function $u_{0}$ which satisfies $N\left(\nabla u_{0}\right) \in$ $L^{p}\left(\Sigma_{T}\right)$. Furthermore, we can choose the subsequence so that the convergence is uniform on each $\Omega_{\eta, T}$ for $\eta>0$. Applying the estimate of Lemma 1.3 to $u_{i}-u_{j}$, it follows that $\left\|N\left(\nabla\left(u_{i}-u_{j}\right)\right)\right\|_{L^{p}\left(\Sigma_{T}\right)} \rightarrow 0$ as $i, j \rightarrow \infty$. This, (5.3) and the uniform convergence of the $\alpha_{i}$ imply that $\partial_{\alpha_{0}} u_{0}=0$ on $\Sigma_{T}$. Hence, Proposition 5.1 implies that $u_{0}=0$. On the other hand, Lemma 1.3, (5.3) and (5.4) imply that $\left\|\nabla u_{i}\right\|_{L^{p}\left(\Omega_{\eta, T}\right)} \geq c>0$. This and the uniform convergence of $\nabla u_{i}$ on $\Omega_{\eta, T}$ imply that $\left\|\nabla u_{0}\right\|_{L^{p}\left(\Omega_{\eta, T}\right)} \geq c$. This contradicts our previous assertion that $u_{0} \equiv 0$ and establishes the proposition.

Finally, we are ready to establish the existence of solutions to (ODP). Given the a priori estimate of the previous proposition, all we need to do is establish existence for data in a dense subset of $L^{p}\left(\Sigma_{T}\right)$. G. Lieberman has already done this for us and we quote a special case of [L2, Theorem 14.5].

To state Lieberman's result, we recall the definition of parabolic Hölder spaces, $H_{\beta}(\mathscr{O})$ when $0<\beta<1$ and $\mathscr{O} \subset \mathbf{R} \times \mathbf{R}^{n}$. A function $f$ is $H_{\beta}(\mathscr{O})$ if and only if

$$
|f(X)-f(Y)| \leq C \delta(X ; Y)^{\beta}, \quad X, Y \in \mathscr{O}
$$

for some finite constant.

Proposition 5.5 [L2, Theorem 14.5]. Suppose that $\alpha$ is a oblique vector field which is Hölder continuous of exponent $\beta>0$ on $\Sigma_{T}$ and let $f \in H_{\beta}\left(\Sigma_{T}\right) \cap$ $\{f: f(0, p)=0\}$. There exists $\beta_{0}>0$ such that if $\beta<\beta_{0}$, then there exists a solution of $(O D P)$ with datum $f$ and this solution satisfies $\nabla u \in H_{\beta}\left(\Sigma_{T}\right)$. In particular, $\nabla u$ is bounded on $\Omega_{T}$.

Lieberman's Theorem 14.5 gives existence of a solution which lies in a weighted Hölder space denoted by $H_{a}^{(-1-\beta)}\left(\Omega_{T}\right)$ with $a>2$. To extract Proposition 5.5 from his result, observe that $H_{a}^{(-1-\beta)}\left(\Omega_{T}\right) \subset H_{1+\beta}^{(-1-\beta)}\left(\Omega_{T}\right)=$ $H_{1+\beta}\left(\Omega_{T}\right)$ and that functions in $H_{1+\beta}\left(\Omega_{T}\right)$ satisfy $\nabla u \in H_{\beta}\left(\Omega_{T}\right)$. See [L1, pp. 3-4] for definitions and the inclusion. 
Finally, we state and prove our existence result. To do this, it will be convenient to use the space

$$
H^{p}\left(\Omega_{T}\right) \equiv\left\{u: u \text { is caloric in } \Omega_{T}, u(0, x)=0 \text { and } N(\nabla u) \in L^{p}\left(\Sigma_{T}\right)\right\} .
$$

It is easy to see that under the norm

$$
\|u\|_{H^{p}\left(\Omega_{T}\right)} \equiv\|N(\nabla u)\|_{L^{p}\left(\Sigma_{T}\right)},
$$

$H^{p}\left(\Omega_{T}\right)$ becomes a Banach space. Letting $\partial_{\alpha}: H^{p}\left(\Omega_{T}\right) \rightarrow L^{p}\left(\Sigma_{T}\right)$ denote the map $u \rightarrow \partial_{\alpha} u$, what we wish to show is:

Proposition 5.6. Let $\alpha$ be a continuous oblique vector field and let $p$ satisfy $2-\epsilon<p<\infty$. Then the map $\partial_{\alpha}: H^{p}\left(\Omega_{T}\right) \rightarrow L^{p}\left(\Sigma_{T}\right)$ is surjective.

Proof. We first prove this under the assumption that $\alpha$ is Hölder continuous on $\Sigma_{T}$. Then Propositions 5.2 and 5.5 and a routine limiting argument imply that $\partial_{\alpha}$ is surjective since $H_{\beta}\left(\Sigma_{T}\right) \cap\{f: f(0, p)=0\}$ is dense in $L^{p}\left(\Sigma_{T}\right)$.

To remove the restriction that $\alpha$ is Hölder continuous, we use the method of continuity. Let $\alpha_{0}$ be a Hölder continuous oblique vector field and let $\alpha_{1}$ be an arbitrary continuous oblique vector field. For $0<t<1$, define $\bar{\alpha}_{t}=$ $(1-t) \alpha_{0}+t \alpha_{1}$ and $\alpha_{t}=\bar{\alpha}_{t} /\left|\bar{\alpha}_{t}\right|$. From Proposition 5.2, we have that

$$
\|u\|_{H^{p}\left(\Omega_{T}\right)} \leq C\left\|\partial_{\alpha_{t}} u\right\|_{L^{p}\left(\Sigma_{T}\right)}, \quad 0 \leq t \leq 1 .
$$

The previous paragraph implies that $\partial_{\alpha_{0}}$ is surjective, hence the method of continuity implies that $\partial_{\alpha_{1}}$ is also surjective.

This proposition completes the proof of the Theorem. Uniqueness was established in Proposition 5.1 and the estimate of the Theorem was given in Proposition 5.2.

\section{REFERENCES}

[B1] R. M. Brown, Layer potentials and boundary value problems for the heat equation on Lipschitz cylinders, Thesis, University of Minnesota, 1987.

[B2] - Area integral estimates for caloric functions, Trans. Amer. Math. Soc. (to appear).

[B3] The initial-Neumann problem for the heat equation in Lipschitz cylinders, Trans. Amer. Math. Soc. (to appear).

[C] A. P. Calderón, Boundary value problems in Lipschitzian domains, Recent Progress in Fourier Analysis, Elsevier Science Publishers, 1985, 33-48.

[CF] R. R. Coifman and C. Fefferman, Weighted norm inequalities for maximal functions and singular integrals, Studia Math. 51 (1974), 241-250.

[FS] E. B. Fabes and S. Salsa, Estimates of caloric measure and the initial-Dirichlet problem for the heat equation in Lipschitz cylinders, Trans. Amer. Math. Soc. 279 (1983), 635-650.

[JK] D. S. Jerison and C. E. Kenig, Boundary value problems in Lipschitz domains, Studies in partial differential equations, MAA Studies in Mathematics,, v. 23, Math. Assoc. Amer., Washington, D.C., 1982, 1-68.

[K] J. Kemper, Temperatures in several variables: Kernel functions, representations and parabolic boundary values, Trans. Amer. Math. Soc. 167 (1972), 243-262.

[KP] C. E. Kenig and J. Pipher, The oblique derivative problem on Lipschitz domains with $L^{p}$ data, Amer. J. Math. 110 (1988), 715-737. 
[L1] G. Lieberman, Intermediate Schauder theory for second order parabolic equations. I, Estimates, J. Differential Equations. 63 (1986), 1-31.

[L2] _ Intermediate Schauder theory for second order parabolic equations. III, The tusk condition. preprint.

[S] E. M. Stein, Singular integrals and differentiability properties of functions, Princeton University Press, Princeton, NJ, 1970.

[T] A. Torchinsky, Real variable methods in harmonic analysis, Academic Press, 1986.

[Tr] N. S. Trudinger, Pointwise estimates and quasilinear parabolic equations, Comm. Pure Appl. Math. 21 (1968), 205-226.

Department of Mathematics, University of Chicago, Chicago Illinois 60637 